\title{
ELECTRONIC ENERGY TRANSFER IN MIXED ORGANIC SOLIDS: ANDERSON LOCALIZATION OR DELOCALIZATION? *
}

\author{
Eric M. MONBERG ${ }^{*}$ and Raoul KOPELMAN \\ Department of Chemistry, University of Michigan, \\ Ann Arbor, Mfichigan 48109, USA
}

Received 26 May 1978

Revised manuscript received 15 July 1978

\begin{abstract}
A simple Anderson transition model, ignoring guest clusterization, excitation lifetime, sensor concentration, excitonphonon coupling and thermalization, appears to be incompatible with the critical concentrations observed for triplet exciton transport in several ternary crystal systems. Dynamic percolation, involving hopping or tunneling through longrange clusters, remains our suggested model.
\end{abstract}

Recent experimental studies of electronic energy transfer in isotopic mixed naphthalene $[1-4]$ and benzene [5-7] crystals at low temperatures have established the existence of a "critical" concentration of the isotopic guest below which energy migration is abruptly reduced. This phenomenon has been attributed to "exciton percolation", a concept embracing a number of specific models [8], including static and dynamic clusters $[9,4]$ as well as stochastic and quasi-coherent propagation [10]. While Anderson localization can play a role in percolation models [8], diagnol Anderson localization has been very recently suggested as the only pertinent factor, to the exclusion of the percolation and/or scaling concepts [11]. Specifically, Klafter and Jortner [11] (KJ) fitted the critical concentrations of the triplet exciton migration in ternary naphthalene systems $[1,2]$ with a superlattice model, deriving therefrom approximate values for the inhomogeneous broadening in those systems. The purpose of this comment is to argue against the $\mathrm{KJ}$ interpretation for these specific systems, and thereby elucidate the factors that have to be considered when studying the energy migration in any such systems. Specifically, we dwell on the importance of guest

\footnotetext{
- Supported by NIH Grant NS08116-08.

* Present address: Western Electric, Engineering Research Center, Princeton, New Jersey 08540, USA.
}

clusterization, excitation lifetime and exciton-phonon interactions, which are not included in the $\mathrm{KJ}$ model.

According to the formalism of KJ (Klafter and Jortner) one expects an Anderson localization whenever (roughly)

$$
\delta>2.8 B \quad(B=2 Z n)
$$

where $\delta$ is the diagonal inhomogeneity and $J$ the exciton band parameter given by [11]

$J \approx \beta^{n} / \Delta^{n-1}$.

Here, as usual [12], $\beta$ is the dominant exciton pairwise interaction, $\Delta$ the trap depth (guest-host energy separation) and $n$ the number of nearest neighbor bonds connecting two guest sites. $\mathrm{KJ}$ used the well known $[13,14]$ value of $\beta=1.25 \mathrm{~cm}^{-1}$ for this system. It is also agreed that the longer range direct pairwise interactions contribute little since these smaller interactions [14-16] only affect eq. (2) through their $n$th powers $[2,5]$. $\mathrm{KJ}$ also used the preciseiy known [17] values of $\Delta$. We estimate $Z=2$ (and certainly $Z<4$ ). The crucial parameter is the number $n$, not only because its effect is obviously "exponential", but because a correct handling of the statistics involves lattice percolation, i.e., cluster statistics $\$$. The accepted

F In ref. [11] an "averaged" density of guests was used, implying a superlattice and ignoring the random correlations responsible for the formation of clusters. 
$[2,5,9,18]$ idea, with regard to this point, is that at a given guest (i.e., $\mathrm{C}_{10} \mathrm{H}_{8}$ ) concentration one uses an effective guest-guest bond distance. The latter has to be defined correctly, so as to identify a guest-guestguest... connected path [19] that will enable significant exciton transfer. In order to have an even probability to register at a supertrap (i.e., $\beta$-methylnaphthalene) whose relative concentration with respect to the trap is 1 mole \%, such a connected path has to include a minimum average number of about $10^{2}$ guest sites. The accepted quantitative methods of identifying such a connected-guest-cluster fall within the cluster distribution enumerations used in modern percolation theory $[9,19,20]$. We note that the critical concentration $C_{\mathrm{c}}$ for forming a 100 -ciuster or a 1000 -cluster is very close to that for an "infinite" cluster [9]. To improve on the simple averaging (superlattice model) two independent methods have been devised within the last couple of years $[1,2]$ to evaluate $C_{\mathrm{c}}$ as a function of $n$, which give mutually consistent results. We now work "backwards" and get, for a given concentration, the minimum value of $n$ sufficient to establish connectivity (lattice site percolation). Taking a concentration of about 7 mole \% one obtains (from fig. ? of ref. [2]), for a two-dimensional square lattice topology, a value of $n=5$. We thus calculate, from eq. (2), $J=2 \times 10^{-8}$ $\mathrm{cm}^{-1}$. As we observe experimentally for the $\mathrm{C}_{10} \mathrm{H}_{8} i$ $C_{10} D_{8}$ system a significant rise in exciton transfer at about 7 or $8 \%$, we must conclude, according to Ylafter and Jortner [5], i.e. eq. (1), that our diagonal inhomogeneity is $\delta \leqslant 2 \times 10^{-7} \mathrm{~cm}^{-1}$. As experimentalists, we would consider it a compliment to have produced samples that are this homogeneous (at least. on a domain level). However, we believe that such a compliment is not necessarily justified, for the reasons given below.

By plugging into eq. (2) somewhat questionable values of $n$ (as they ignored clusterization), Klafter and Jortner have fitted some of our previous data [2], using $\delta$ as a parameter to force eq. (1) into a rough equality. This procedure, in principle, should give the correct order of magnitude if diagonal Anderson localization is involved. They assumed, however, that for each series of samples having a common isotopic trap depth $\Delta$, there was a common value of $\delta$, which was different by an order of magnitude or more from the $\delta$ of a different series. For the reasons given above, we not only doubt their particular range of values of $\delta$
( 0.03 to $\left.3 \mathrm{~cm}^{-1}\right)$, but we contest their basic assumption which requires different inhomogeneous line broadenings for each trap depth. We see no justification for treating all the samples within a series as having the same $\delta$ [actually as small as $10^{-9} \mathrm{~cm}^{-1}$ according to eqs. (1) and (2)] while letting sets of samples with different $\Delta$ have different $\delta$ values. This does not mean that a given isotopic substitution could not infiuence $\delta$ (in a similar way to the "orientation splitting" we have observed) $[17,22]$, however, we do not see any reasonable correlation between $\Delta$ (primarily a molecular effect [12]) and $\delta$ (a crystal field fluctuation [23]). In contrast, our earlier theoretical approach $[1,2,21]$ predicts a monotonic behavior with $\Delta$ for both $n$ and $C_{c}$, as is indeed observed.

We notice that at 8.3 mole \% of guest, according to table 2 of ref. [3], about $20 \%$ of the guest molecules form nearest neighbor pairs, about $7 \%$ form trimers, $2 \%$ tetramers, etc. At $17 \%$ slightly more than half the guest molecules are still distributed as dimers, trimers, tetramers (of various shapes and energy levels) [24], etc. while less than half are "monomers" (still ignoring longer range pairs [15]). As the monomer-dimer energy separation is $1.25 \mathrm{~cm}^{-1}$ and the dimer-trimer separation is $0.5 \mathrm{~cm}^{-1}$ and so on (exact relative values are given in ref. [24]), we get an effective energy fluctuation $\delta^{\prime}$, which is much larger than the $J$ values at these concentrations (i.e., $\gg 10^{-4} \mathrm{~cm}^{-1}$ ). One might then ask why is there considerable exciton transfer at these concentrations? Why should not such an "offdiagonal" Anderson fluctuation $\left(\delta^{\prime}\right)$ break up. "the band", cause localization and stop the exciton conduction ? Evidently it does not, and we have discussed in great detail the reason for this $[2-4,21]$ in terms

\footnotetext{
+ Those are their four adjustable parameters "w" with which they fitted our experimental critical concentrations [2]. In contrast, our earlier approach [2,21] only used one adjustable parameter. It should also be noted that the Anderson model implies an exciton-phonon interaction smaller than " $w$ ". Thus, for consistency, one has to use the band model, rather than the hopping model, for $C>C_{\mathrm{C}}$.

$\ddagger$ We note that $\delta^{\circ} \approx \beta$ and thus an off-diagonal Anderson localization [25], if defined in this manner (i.e., the energy fluctuations are just due to cluster splittings), occurs whenever $\beta>J$. This condition is met as soon as $\pi>1$, i.e., just below the static percolation concentration [3]. We emphasize here that the above is not the usual definition [26] of "off-diagonal Anderson localization".
} 
of thermalization, giving a necessary condition for extensive transfer:

$\delta^{\prime} \lesssim k T$.

Further experiments bearing on the thermalization effects have been carried out on the very same samples $[21,27,28]$. They all indicate that exciton transfer is extensive above $C_{c}$ as soon as eq. (3) is valid. We have pointed out in the past $[2,4]$ that one would require experimental temperatures of about $0.1 \mathrm{~K}$ or less in order to see effects due to such "off-diagonal" Anderson localization in our samples. The same, presumably, is true for diagonal Anderson localization i.e., the Klafter-Jortner model. We also note that the Klafter-Jortner model is insensitive to the exciton lifetime and supertrap concentrations. These parameters are of prime importance in the dynamic percolation model [2]. (Preliminary results [29] show a strong time dependence for the triplet system in contrast to the model of ref. [11].) Our recent experimental studies have also shown, for naphthalene [21], that both singlet and triplet critical concentrations are consistent with the same dynamic percolation model [21]. Likewise, the large qualitative differences between the singlet and triplet critical concentrations for the exciton transport in benzene [5] cannot be rationalized in terms of an Anderson transition as it is known that the parameters $\beta$ are quite similar for the singlet and triplet states. One also does not expect the diagonal fluctuations $\delta$ to differ significantly from the singlet to the triplet [5-7]. The basic difference between the singlet and triplet cases is the enormous difference in exciton lifetime (nanoseconds versus seconds). The long triplet lifetimes allow clustercluster jumps that are more extended both in space and in time and lead to a lower critical dynamic percolation concentration for the triplet systems.

We notice that Mott's criticism [30,31] of the use of "classical" percolation-type theory for certain kinds of electron conduction-to-insulator transitions $[32,33]$ has been linited to the case of ordinary conduction bands. However, in the case of electron conduction via hopping and/or tunneling, involving thermal activation, as first pointed out by Miller and Abrahams [34], Mott seems to concur ${ }^{\ddagger}$ with the ap-

* Ref. [31], p. 34. Note also the analogy of our case with the "hopping integral" connectivity (percolation) model for metal-insulator transitions in Cu : Ar films (ref. [30], p. 627). plication of percolation theory to the problem of the most favored paths. We believe that in most, if not all, the traditional examples of long-range exciton trap-totrap migration [2,5], the energy transfer process is much closer to the limit of hopping [2] via "virtual" bands [2] rather than coherent exciton motion via ordinary bands which assumes little phonon and/or defect scattering. We thus believe that our exciton transport experiments should be related to what Mott called the variable range hopping regime, which is very different from the regime of the Mott-Anderson transition $[30,33]$. It is thus a regime of exciton percolation via hopping which is analogous to exciton diffusion via hopping in a perfect crystal.

In conclusion, while classical electron percolation [35,36] requires metallic channels of "classical" dimensions ${ }^{\neq}$, involving long-range fluctuations in the composition of a disordered material [30], the cluster model of exciton percolation $[4,8]$ iequires only chains of molecules on the scale of the crystalline lattice constant. Also, our triplet exciton conduction is operationally defined by our experiments to be on a molecular scale, or, at most, within a microdomain *. A "chain of transfer" is likely to have a typical "diameter" of only one or a few lattice constants [37]. The occurrence in principle of an Anderson-Mott type transition [31] for exciton transfer within such a microdomain is questionable $[38,39]$. In contrast, the microscopic percolation model $[2,4]$ appears to be most appropriate for describing the phonon assisted [40] exciton tunneling (or hopping) within such domains. It also seems to be agreed now [41] that a dynamic (time dependent) model of exciton percolation $\frac{}{+}$ is more appropriate than an Anderson localization model for some of our naphthalene singlet exciton transport experiments $[3,4,10]$. Thus, even if the Anderson localization model is valid, within the appropriate domains, for $k T \ll \delta^{\prime}$, i.e., at very low temperatures, we suggest that the excitations have been

\%ef. [31], p. 35. However, note the effect of finite exciton lifetime.

* I.e., much larger thar an atomic size cross section [30,33].

* This is due to the registration of our exciton transport via randomly dispersed "supertrap" molecules (with a $C_{\mathrm{S}}$ of $10^{-4}$ to $10^{-3}$ ).

I We note that the term "percolation" is a generalization of "diffusion" for heterogeneous media and includes both stochastic and ronstochastic kinetics. 
"delocalized" by interactions with phonons and are thus able to percolate.

\section{References}

[1] R. Kopelman, E.M. Monbery, F.W. Ochs and P.N. Prasad, J. Chem. Phys. 62 (1975) 292.

[2] R. Kopelman, E.M. Monberg and F.W. Ochs, Chem. Phys. 19 (1977) 413, and references therein.

[3] R. Kopelman, E.M. Monberg and F.W. Ochs, Chem. Phys. 21 (1977) 373, and references therein.

[4] R. Kopelman, in: Topics in applied physics, Vol. 15 , ed. F.K. Fong (Springer, Berlin, 1976) p. 298; J. Luminessence 12/13 (1976) 775; J. Phys. Chem. 80 (1976) 2191.

[5] S.D. Colson, S. George, T. Keyes and V. Vaida, J. Chem. Phys. 67 (1977) 4941, and references therein.

[6] S.D. Colson, R.E. Tumer and V. Vaida, J. Chem. Phys. 66 (1977) 2187.

[7] F.B. Tudron and S.D. Colson, J. Chem. Phys. 65 (1976) 4184.

[81 J. Hoshen and R. Kopelman, J. Chem. Phys 65 (1976) 2817;

E.M. Monberg and R. Kopelman, Chem. Phys. Letters 58 (1978) 492.

[9] J. Hoshen, EM. Monberg and R. Kopelman, I. Stat. Phys, to be publisted.

[10] P. Argyrakis and R. Kopelman, J. Chem. Phys. 66 (1977) 3301.

[11] J. Klafter and J. Joxtner, Chem. Phys. Letters 49 (1977) 410.

[12] R. Kopeiman, in: Excited states, Vol 2, ed. E.C. Lim (Academic Press, New York, 1975) p. 33.

[13] B.I. Botter, C.I. Nonhof, J. Schmidt and J.H. van der Waals, Chem. Phys. Lettzrs 43 (1976) 210.

[14] H.C. Wolf, J. Luminescence 12/13 (1976) 33; D.M. Hanson, J. Chem. Phys. 52 (1970) 3409.

[15] B.J. Botter, A.J. vaz Strien and J. Schmidt, Chem. Phys Letters 49 (1977) 39.

[16] L. Altweg, M. Chaber and I. Zschokke-Granacher, Fhys. Rev. B14 (1976) 1963. [17i F.W. Ochs, P.N. Prasad and R. Kopelman, Chem. Phys. 6 (1974) 253.

[18] M. Pollaik, J. Noncryst. Solids 11 (1972) 1; M. Pollak, M.I. Knotek, M.L. Kurtsman and H. Glick, Phys. Rev. Letters 30 (1973) 856.

[19] D. Stauffer, Z. Physik B25 (1976) 391.

[20] C. Domb and M.S. Green, Fuase transitions and critical phenomena (Academic Press, New York, 1972) p. 224.

[21] E.M. Monberg, Ph.D. Thesis, University of Michigan (1977).

[22] F.W. Ochs, Ph.D. Thesis, University of Michigan (1974).

[23] F.W. Anderson, Phys. Rev. 109 (1958) 1492.

[24] J. Hoshen and R. Kopelman, Phys. Stat. Sol. $81 \mathrm{~b}$ (1977) 479.

[25] P.D. Smith, R.D. Mead and A.H. Zewail, Chem. Phys. Letters 50 (1977) 358.

[26] P.D. Antoniou and E.N. Economou, Phys. Rev. B16 (1977) 3768.

[27] E.M. Monberg and R. Kopelman, unpublished.

[28] P.N. Prasad, F.W. Ochs and R. Kopelman, unpublished.

[29] D. Ahlgren and R. Kopelman, unpublished.

[30] N.F. Mott, Phil. Mag. 29 (1974) 613; Comments Phys. 1 (1976) 203.

[31] N.F. Mott, Metal-insulator transitions (Taylor and Francis, London, 1974).

[32] M.H. Cohen and J. Jortner, Phys. Rey. Letters 30 (1973) $696,699$.

[33] E.N. Economou and M.H. Cohen, Phys. Rev. B5 (1972) 2931.

[34] A. Miller and E. Abrahams, Phys. Rev. 120 (1960) 745.

[35] B.J. Last and D.J. Thouless, Phys. Rev. Letters 27 (1971) 1719.

[36] S. Kirkpatrick, Rev. Mod. Phys. 45 (1973) 574.

[37] R. Kopelman, Rec. Chem. Progr. 31 (1970) 211.

[38] S.D. Colsón and R. Kopelman, unpublished.

[39] D.J. Thouless, Phys. Rev. Letters 39 (1977) 1167.

[40] T. Holstein, S.K. Lyo and R. Orbach, Phys. Rev. Letters 36 (1976) 891; Phys. Rev. B15 (1977) 4693; B16 (1977) 934.

[41] J. Jortner, private communication (April 1978). 